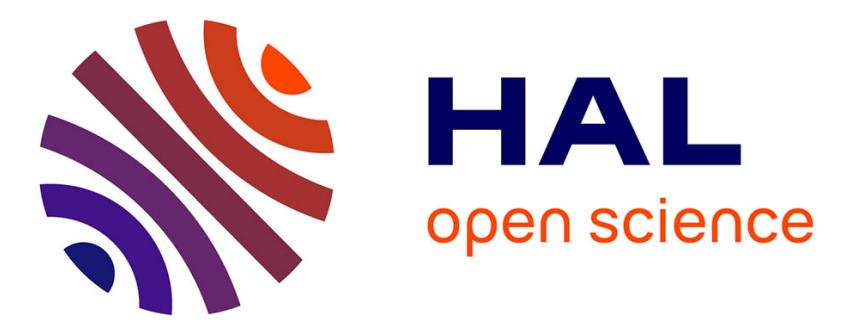

\title{
Joint Stiffness Identification of Industrial Serial Robots
}

Claire Dumas, Stéphane Caro, Cherif Mehdi, Sébastien Garnier, Benoît Furet

\section{To cite this version:}

Claire Dumas, Stéphane Caro, Cherif Mehdi, Sébastien Garnier, Benoît Furet. Joint Stiffness Identification of Industrial Serial Robots. Robotica, 2011, pp.1-20. 10.1017/S0263574711000932 . hal00633095

\section{HAL Id: hal-00633095 \\ https://hal.science/hal-00633095}

Submitted on 17 Oct 2011

HAL is a multi-disciplinary open access archive for the deposit and dissemination of scientific research documents, whether they are published or not. The documents may come from teaching and research institutions in France or abroad, or from public or private research centers.
L'archive ouverte pluridisciplinaire HAL, est destinée au dépôt et à la diffusion de documents scientifiques de niveau recherche, publiés ou non, émanant des établissements d'enseignement et de recherche français ou étrangers, des laboratoires publics ou privés. 


\title{
Joint Stiffness Identification of Industrial Serial Robots
}

\author{
Claire Dumas $^{a *}$, Stéphane Caro $^{a}$, Mehdi Cherif $^{b}$, Sébastien Garnier ${ }^{a}$, Benoît Furet $^{a}$ \\ ${ }^{a}$ Institut de Recherche en Communications et Cybernétique de Nantes, \\ UMR CNRS n 6597, 1 rue de la Noë, 44321 Nantes, France \\ ${ }^{b}$ Laboratoire de Génie Mécanique et Matériaux de Bordeaux, \\ 15 rue Naudet CS 10207, 33175 Gradignan Cedex
}

\begin{abstract}
This paper presents a new methodology for the joint stiffness identification of industrial serial robots and as consequence for the evaluation of both translational and rotational displacements of the robot's end-effector subject to an external wrench (force and torque). In this paper, the robot's links are supposed to be quite stiffer than the actuated joints as it is usually the case with industrial serial robots. The robustness of the identification method and the sensitivity of the results to measurement errors and number of experimental tests are also analyzed. The Kuka KR240-2 robot is used as an illustrative example throughout the paper.
\end{abstract}

Keywords: Stiffness Analysis; Joint Stiffness Identification; Serial Robots.

\section{Nomenclature}

$\begin{array}{ll}L_{i=0 . .6} & \text { Links }\left(L_{0} \text { is the base and } L_{6} \text { is the end-effector) }\right. \\ \mathcal{F}_{0} & \text { Frame attached to the base according to Denavit Hartenberg modified convention } \\ \mathcal{F}_{i=1 . .6} & \text { Frames attached to the moving links according to Denavit Hartenberg modified convention } \\ O_{i=1 . .6} & \text { Origin of frame } \mathcal{F}_{i} \\ \mathbf{x}_{i=1 . .6} & \mathbf{x} \text { axis of frame } \mathcal{F}_{i} \\ \mathbf{y}_{i=1 . .6} & \mathbf{y} \text { axis of frame } \mathcal{F}_{i} \\ \mathbf{z}_{i=1 . .6} & \mathbf{z} \text { axis of frame } \mathcal{F}_{i} \\ \theta_{i=1 . .6} & \text { Actuated joint angles } \\ \mathbf{J} & \text { Kinematic Jacobian matrix of the robot } \\ \mathbf{K}_{C} & \text { Complementary Stiffness matrix of the robot } \\ \mathbf{K}_{X} & \text { Cartesian Stiffness Matrix of the robot } \\ \mathbf{K}_{\theta} & \text { Joint stiffness matrix } \\ k_{\theta_{i=1 . .6}} & \text { Joint stiffness values } \\ \delta \mathbf{d} & \text { Small displacement screw of the robot's end-effector expressed in } \mathcal{F}_{0} \\ \dot{\mathbf{p}} & \text { End-effector translational velocity vector expressed in } \mathcal{F}_{0} \\ \boldsymbol{\omega} & \text { End-effector angular velocity vector expressed in } \mathcal{F}_{0} \\ \mathbf{t} & \text { End-effector twist expressed in } \mathcal{F}_{0} \\ \boldsymbol{w} & \text { Wrench applied to the robot's end-effector expressed in } \mathcal{F}_{0} \\ Z_{i c}(i=1,2,3) & \text { Zones of the joint space }\left(\theta_{2}, \theta_{3}\right) \text { where the robot has a good dexterity } \\ Z_{\nu_{p}} & \text { Zone where the influence of } \mathbf{K}_{C} \text { on } \mathbf{K}_{\theta} \text { is at its minimum with regard to } \nu_{p} \\ Z_{\nu_{r}} & \text { Zone where the influence of } \mathbf{K}_{C} \text { on } \mathbf{K}_{\theta} \text { is at its minimum with regard to } \nu_{r} \\ \end{array}$

\footnotetext{
${ }^{*}$ Corresponding author. Email address: claire.dumas@irccyn.ec-nantes.fr
} 


\section{Introduction}

Serial robots are mainly used in industry for tasks that require a good repeatability, but not necessarily good global pose accuracy (position + orientation as defined in ISO9283) of the robot's end-effector (EE). For example, these robots are generally used for pick-and-place, painting and welding operations. Nevertheless, they are now being used for machining operations such as the trimming, deflashing, degating, sanding and sawing of composites parts that require high precision and stiffness. Therefore, to perform these operations, the robots must show good kinematic and elastostatic performance. In this context, it appears that conventional machine tools such as the gantry CNC, are still more efficient than serial robots. That is the reason why it is relevant to pay attention to the robots performance in order to optimize their use for machining operations. Some research works discuss the following:: (i) the tool path optimization considering both kinematic and dynamic robot performance $[1,2,3]$; (ii) the determination of optimal cutting parameters to avoid tool chattering [3, 4]; (iii) the robot stiffness analysis [5]; and (iv) the determination of robot performance indices [6, 7, 8, 9, 10]. Robot stiffness is also a relevant performance index for robot machining [11]. Accordingly, this paper discusses the stiffness modelling of serial robots as well as the identification of their stiffness parameters. As a matter of fact, some stiffness models exist in the literature for serial and parallel manipulators [12, 13]. However, the identification of their stiffness parameters has yet to be determined.

A model based on the conservative congruence transformation (CCT), introduced in [14], was used in [15] to identify the stiffness values of the first three actuated revolute joints of a $6 \mathrm{R}$ robot. This model was used instead of the common formula developed in [16], which is only valid for unloaded manipulators. Only the robot translational part, i.e. the three first joint stiffness values, are identified. Two methods were presented in [17] to obtain the Cartesian stiffness matrix (CaSM) of a 5R robot. The first method consists of clamping all the joints except one to measure its stiffness and repeating the procedure for each joint. As a result, only five experiments are useful to evaluate the CaSM of the robot throughout its Cartesian workspace if the link stiffnesses are known. However, to clamp the joints is not an easy task and the link stiffnesses are not usually known. The second method consists of measuring the deflection of the robot due to some loads exerted on its end-effector and evaluating its stiffness throughout its Cartesian workspace by means of some interpolations. This method provides better results, but many configurations have to be tested in order to get a good approximation of the CaSM of the robot throughout its Cartesian workspace. The results are better with this method because the link stiffnesses are considered in addition to the joint stiffnesses. Indeed, when a load is applied to the robot end-effector, all deformations are considered including those due to the links deformations and/or radial joint stiffness values. Pham et al. [18] proposed a method for the identification of joint stiffness with bandpass filtering and based on the robot's dynamic model. This method requires a closed-loop control in addition to real-time actuator currents.

This paper introduces a method to identify the joint stiffness values of an industrial 6-DOF serial robot. This method aims to evaluate the joint stiffness values of any $6 \mathrm{R}$ serial robot and is based on the conservative congruence transformation [14]. This method is easy to use and fast as it does not require any closed-loop control, nor actuator currents. Besides, the robustness of the method and the sensitivity of the results to measurement errors and to the number of experimental tests are analyzed.

The Kuka KR240-2 robot is used as an illustrative example throughout the paper. Section 2 deals with its kinematic and stiffness modeling. The method proposed for the identification of the joint stiffness values is shown in Section 3. The experimental setup is presented in Section 4. Finally, Section 5 analyzes the robustness of the method and the sensitivity of the results to measurement errors and to the number of experimental tests. 


\section{Kinematic and Stiffness Modelling}

The kinematic and stiffness models used to develop the method for joint stiffness identification are described in this section.

\subsection{Parameterization and Kinematic Modelling}

The modified Denavit Hartenberg (DHm) parameters defined in [19] can be used to parameterize any $6 \mathrm{R}$ serial robot. As illustrated in Fig. 1, the robot is composed of seven links, denoted $L_{0}, \ldots, L_{6}$, and six revolute joints. Link $L_{0}$ is the base of the robot while link $L_{6}$ is the terminal link. Joint $j$ connects link $j$ with link $j-1$, $j=1, \ldots, 6$. Frame $\mathcal{F}_{j}$ attached to link $j$ is defined such that:

- the $\mathbf{z}_{j}$ axis is along joint $j$ axis;

- the $\mathbf{x}_{j}$ axis is along the common normal between $\mathbf{z}_{j}$ and $\mathbf{z}_{j+1}$. If the axes $\mathbf{z}_{j}$ and $\mathbf{z}_{j+1}$ are parallel, the choice of $\mathbf{x}_{j}$ is not unique;

- the origin $O_{j}$ is the intersection of $\mathbf{z}_{j}$ and $\mathbf{x}_{j}$.

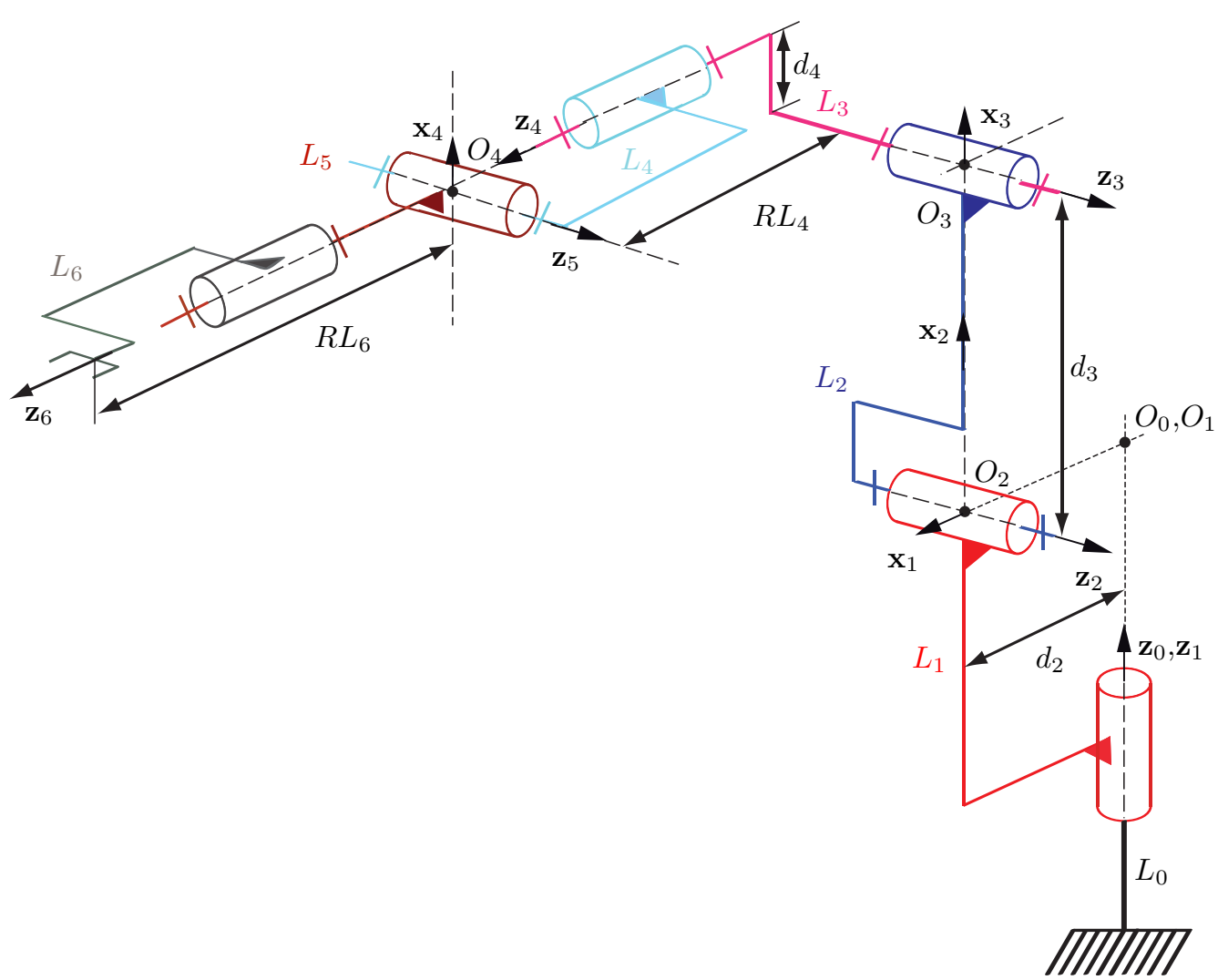

Figure 1: DHm Parameterization of the Kuka KR240-2 robot

The $6 \times 6$ kinematic Jacobian matrix $\mathbf{J}$ of the robot is obtained by means of its DHm parameters and the SYMORO+ software [20] developed in IRCCyN. It relates the instantaneous joint motions to the instant 
Cartesian motions of the end-effector, namely,

$$
\mathbf{t}=\left[\begin{array}{c}
\dot{\mathbf{p}} \\
\boldsymbol{\omega}
\end{array}\right]=\mathbf{J} \dot{\theta}
$$

$\mathbf{t}$ is the end-effector twist expressed in the base frame $\mathcal{F}_{0}$ and composed of its translational velocity vector $\dot{\mathbf{p}}$ and its angular velocity vector $\boldsymbol{\omega}$. Moreover,

$$
\dot{\theta}=\left[\begin{array}{llllll}
\dot{\theta_{1}} & \dot{\theta_{2}} & \dot{\theta_{3}} & \dot{\theta_{4}} & \dot{\theta_{5}} & \dot{\theta_{6}}
\end{array}\right]^{T}
$$

$\dot{\theta}_{i}$ being the $i$ th actuated revolute joint rate.

A closed-form expression of $\mathbf{J}$ and the DHm parameters of the robot are given in Appendix A. The kinematic performance of the robot based on matrix $\mathbf{J}$ are analyzed in Section 3.

\subsection{Stiffness Modelling}

In this paper the robotic-system response to an applied external wrench under static equilibrium is analyzed through the CaSM of the robot. It is possible to determine the translation and angular deflections of the robot EE when it is subjected to an applied wrench.

The robot deflection is due to both its link and joint flexibilities. However, as mentioned in [6, 15], the joint flexibilities are mainly responsible for the global flexibility of any serial robot. Accordingly, in order to come up with a simple stiffness model - also called elastostatic model - of the robot, it is assumed that its links are rigid and its joints are linear elastic torsional springs. As a matter of fact, the simpler the elastostatic model of the robot, the easier the identification of its stiffness parameters. The damping is also supposed to be negligible for a matter of model simplicity.

The relation between the 6 -dimensional vector $\boldsymbol{\Gamma}$ of actuated torques and the corresponding 6 -dimensional vector $\boldsymbol{w}$ of external forces and moments exerted on the robot end-effector is given by:

$$
\boldsymbol{\Gamma}=\mathbf{J}^{T} \boldsymbol{w}
$$

$\boldsymbol{\Gamma}$ can also be expressed as a function of $\delta \boldsymbol{\theta}$, the 6 -dimensional vector of variations in the joint angles, as follows:

$$
\boldsymbol{\Gamma}=\mathbf{K}_{\theta} \delta \boldsymbol{\theta}
$$

with

$$
\mathbf{K}_{\theta}=\left[\begin{array}{cccccc}
k_{\theta_{1}} & 0 & 0 & 0 & 0 & 0 \\
0 & k_{\theta_{2}} & 0 & 0 & 0 & 0 \\
0 & 0 & k_{\theta_{3}} & 0 & 0 & 0 \\
0 & 0 & 0 & k_{\theta_{4}} & 0 & 0 \\
0 & 0 & 0 & 0 & k_{\theta_{5}} & 0 \\
0 & 0 & 0 & 0 & 0 & k_{\theta_{6}}
\end{array}\right]
$$

$k_{\theta_{i}}$ being the $i$ th joint stiffness value. 
The differentiation of Eq. (3) with respect to $\boldsymbol{\theta}$ leads to the following relationship:

$$
\frac{\partial \boldsymbol{\Gamma}}{\partial \theta}=\left(\frac{\partial \mathbf{J}^{T}}{\partial \theta}\right) \boldsymbol{w}+\mathbf{J}^{T} \frac{\partial \boldsymbol{w}}{\partial \mathbf{d}} \frac{\partial \mathbf{d}}{\partial \theta}
$$

Moreover,

$$
\boldsymbol{w}=\mathbf{K}_{X} \delta \mathbf{d}
$$

where $\mathbf{K}_{X}$ is the Cartesian stiffness matrix and $\delta \mathbf{d}$ the 6 -dimensional small displacement screw of the robot EE.

From Eqs. (4)-(7), we obtain the following expression:

$$
\mathbf{K}_{\theta}=\mathbf{K}_{C}+\mathbf{J}^{T} \mathbf{K}_{X} \mathbf{J}
$$

with

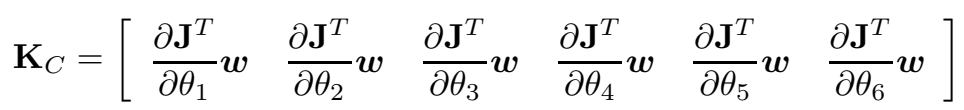

being the complementary stiffness matrix $\mathbf{K}_{C^{-}}$CoSM-defined in [14].

That amounts to

$$
\mathbf{K}_{X}=\mathbf{J}^{-T}\left(\mathbf{K}_{\theta}-\mathbf{K}_{C}\right) \mathbf{J}^{-1}
$$

\section{Method for the Joint Stiffness Identification}

The method proposed for the joint stiffness identification is illustrated in Fig. 2. First, the zones of the robot workspace and joint space in which the robot has a good dexterity are identified. It appears that a good dexterity is required for a good convergence of the procedure. Then, the areas in which $\mathbf{K}_{C}$ is negligible with respect to $\mathbf{K}_{\theta}$ are identified as the stiffness model of the robot can be simplified in those areas. Once good robot configurations are obtained, some of them can be selected in order to perform some tests. The measurement system is illustrated in Section 4.

\subsection{Optimal robot configurations according to kinematic performance}

From Eq. (10), it makes sense that the numerical determination of the joint stiffness values is highly sensitive to the conditioning number of $\mathbf{J}$. As a consequence, the conditioning number of $\mathbf{J}$ is used as a criterion to select appropriate robot configurations for the tests.

In the literature, several kinematic performance criteria have been proposed [21, 22]. Amongst them, the condition number of the Jacobian matrix is widely used to measure the robot dexterity [23].

The condition number $\kappa_{F}(\mathbf{M})$ of a $m \times n$ matrix $\mathbf{M}$, with $m \leq n$, based on the Frobenius norm [24] is defined as follows:

$$
\kappa_{F}(\mathbf{M})=\frac{1}{m} \sqrt{\operatorname{tr}\left(\mathbf{M}^{T} \mathbf{M}\right) \operatorname{tr}\left[\left(\mathbf{M}^{T} \mathbf{M}\right)^{-1}\right]}
$$

This provides an analytical expression of the condition number depending on the posture parameters whereas the 2-norm does not. Besides, it is time efficient to compute matrix inverses rather than singular values. Here, the condition number of matrix $\mathbf{J}$ is meaningless, due to the fact that its terms are not homogeneous; they do not have the same unit. Therefore, as shown in [25] and [26], the Jacobian can be normalized by means of a 


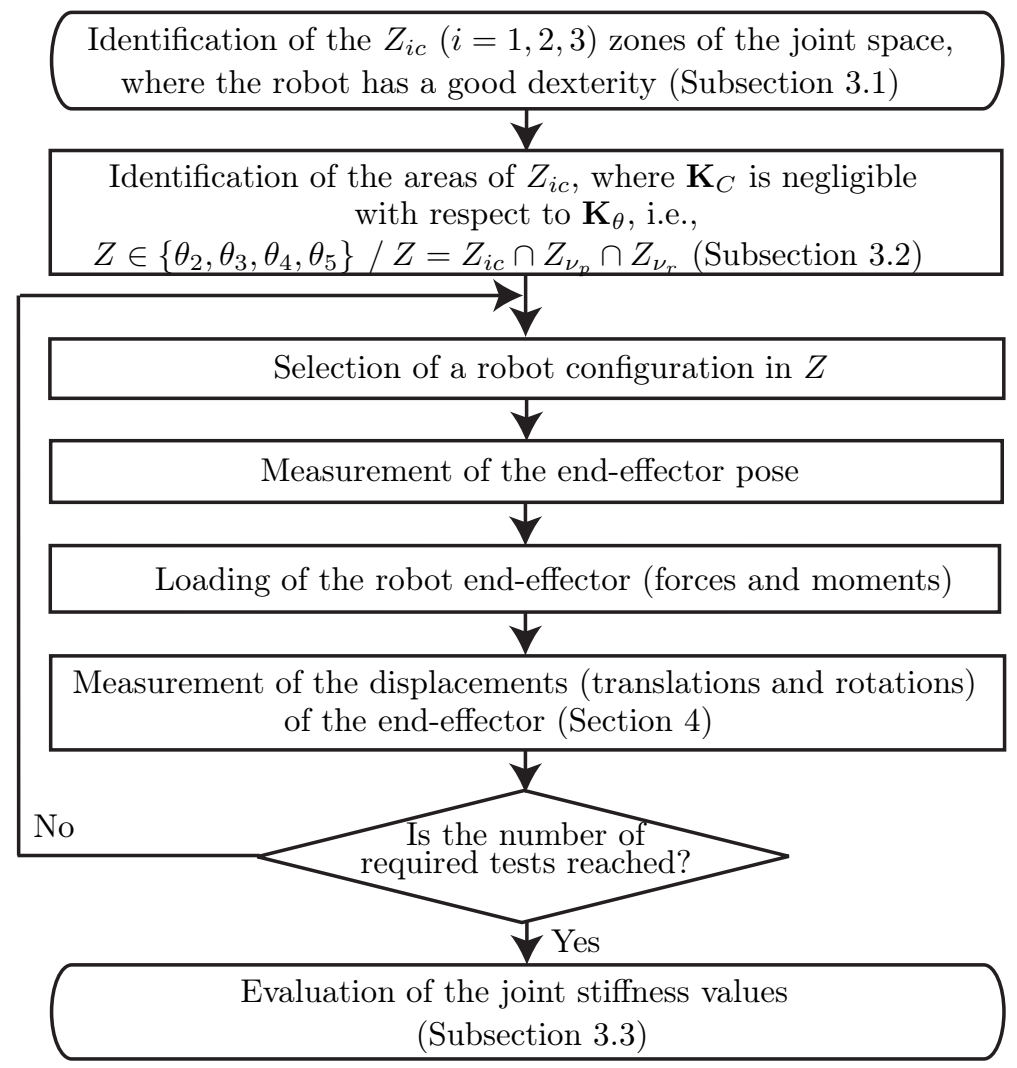

Figure 2: Procedure for the joint stiffness identification

normalizing length. Later on, the concept of characteristic length was introduced in [27] and revisited in [28] in order to avoid the random choice of the normalizing length denoted $L$.

Let $\mathbf{J}_{N}$ be the normalized Jacobian matrix of the Kuka KR240-2 robot expressed as follows:

$$
\mathbf{J}_{N}=\left[\begin{array}{cc}
\frac{1}{L} \mathbf{I}_{3 \times 3} & \mathbf{0} \\
\mathbf{0} & \mathbf{I}_{3 \times 3}
\end{array}\right] \mathbf{J}
$$

$\mathbf{I}_{3 \times 3}$ is the $3 \times 3$ identity matrix and $L$ is the characteristic length of the robot. The characteristic length of the Kuka robot used in this study is equal to 0.682 meter and was obtained by means of the methodology proposed in [28]. It is noteworthy here that the condition number is computed only to identify the zones (on $\theta_{2}$ and $\theta_{3}$ ranges) where the robot has a good dexterity. It appears that the condition number of $\mathbf{J}_{N}$ depends on the characteristic length $L$, but not the location of the zones.

As the second and the third revolute joints are the most influential joints on the translational motions of the end-effector and that the first revolute joint does not affect the robot dexterity, let $\theta_{1}$ be null and the wrist angles $\theta_{4}, \theta_{5}$ and $\theta_{6}$ be set to $45^{\circ}$ so that the corresponding wrist configuration is far from singularities.

Figure 3 depicts the isocontours of the inverse condition number of $\mathbf{J}_{N}$ based on the Frobenius norm, i.e., $\kappa_{F}\left(\mathbf{J}_{N}\right)^{-1}$, throughout the robot Cartesian workspace. The higher $\kappa_{F}\left(\mathbf{J}_{N}\right)^{-1}$, the better the dexterity. On the contrary, the lower $\kappa_{F}\left(\mathbf{J}_{N}\right)^{-1}$, the closer the robot to singularities. Likewise, Fig. 3 shows the isocontours of $\kappa_{F}\left(\mathbf{J}_{N}\right)^{-1}$ throughout the robot joint space. The darkest area corresponds to the nearest positions of the robot to its singularities. The oblique black line characterizes the configurations in which the wrist center is located 


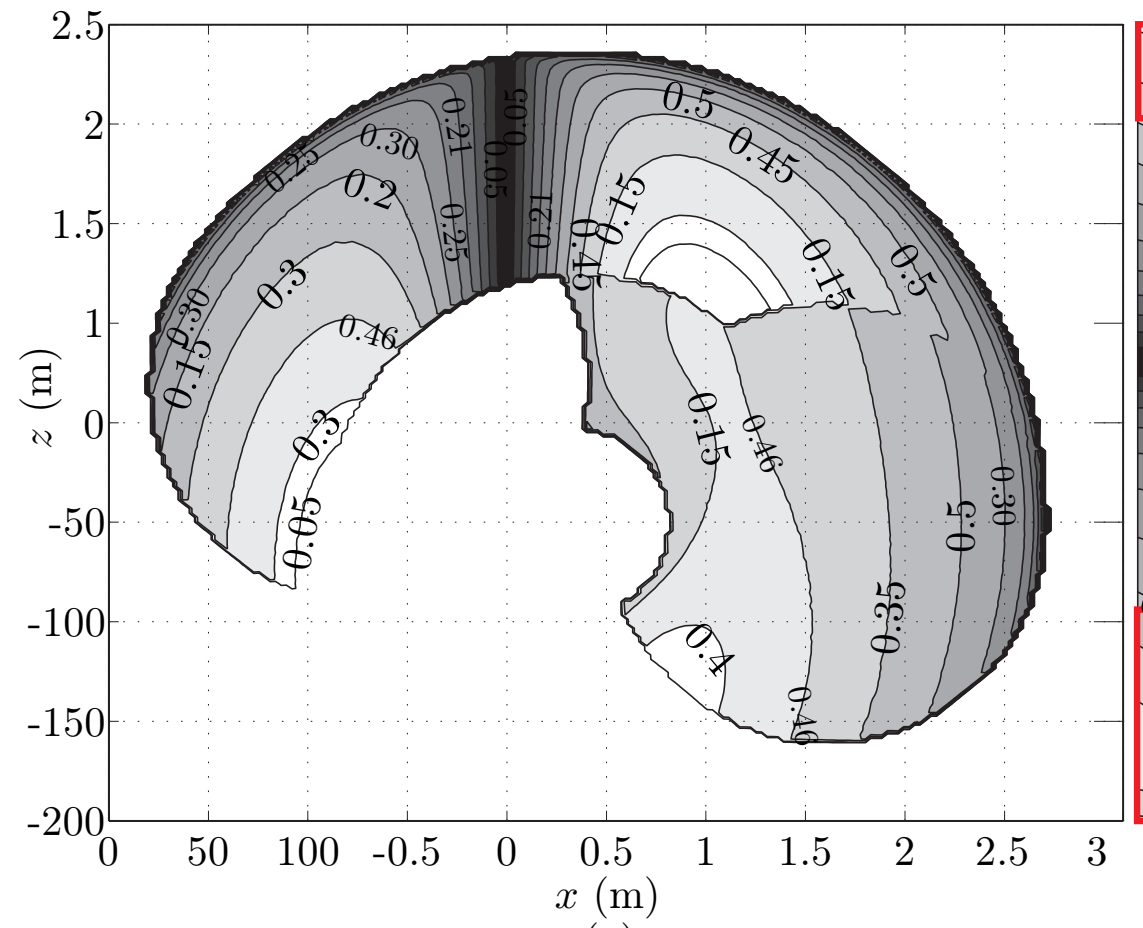

(a)

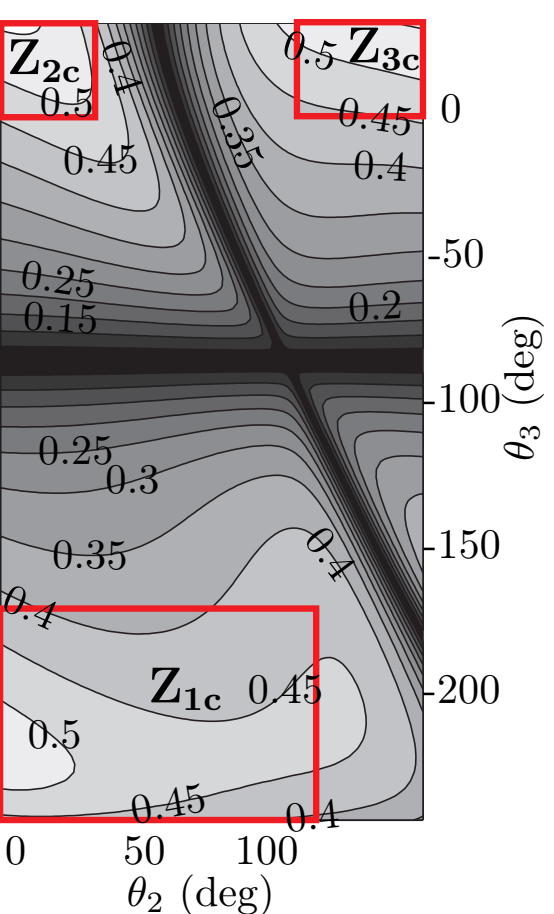

(b)

Figure 3: Contours of the inverse condition number of $\mathbf{J}_{N}$ : (a) in the robot Cartesian workspace and (b) in the joint space $\left(\theta_{2}, \theta_{3}\right)$

on the first joint axis. The horizontal black line in Fig. 3 characterizes the singularities in which the arm is folded.

The choice of appropriate robot configurations for the identification of the joint stiffness values can be made from Figs. 3-(b), namely, $\theta_{2}$ and $\theta_{3}$ should be chosen in the light areas, called $Z_{1 c}, Z_{2 c}$ and $Z_{3 c}$ in Table 1 .

Table 1: Optimal robot configurations defined in its joint space

\begin{tabular}{ccc}
\hline \hline Zone & $\theta_{2}$ & $\theta_{3}$ \\
\hline$Z_{1 c}$ & $0^{\circ}$ to $110^{\circ}$ & $-245^{\circ}$ to $-170^{\circ}$ \\
$Z_{2 c}$ & $0^{\circ}$ to $25^{\circ}$ & $0^{\circ}$ to $29^{\circ}$ \\
$Z_{3 c}$ & $100^{\circ}$ to $146^{\circ}$ & $0^{\circ}$ to $29^{\circ}$ \\
\hline \hline
\end{tabular}

\subsection{Optimal robot configurations according to the influence of $\mathbf{K}_{C}$ on $\mathbf{K}_{X}$}

An optimization algorithm for large-scale nonlinear problems is used in [15] to solve a nonlinear least square optimization problem and the joint stiffness values are determined from Eq. (10). Nevertheless, this method is not robust as it is very sensitive to the starting point of the optimization algorithm. As a consequence, it is relevant to analyze the sensitivity of $\mathbf{K}_{X}$ to $\mathbf{K}_{C}$.

From Eq. (10), $\mathbf{K}_{X}$ depends on both $\mathbf{K}_{\theta}$ and $\mathbf{K}_{C}$. It makes sense that the joint stiffnesses identification is easier when $\mathbf{K}_{C}$ is negligible with respect to $\mathbf{K}_{\theta}$. As a matter of fact, Eq. (10) is reduced to the following equation 
when $\mathbf{K}_{C}$ is negligible with respect to $\mathbf{K}_{\theta}$ :

$$
\mathbf{K}_{X} \approx \mathbf{J}^{-T} \mathbf{K}_{\theta} \mathbf{J}^{-1}
$$

Consequently, the influence of $\mathbf{K}_{C}$ on $\mathbf{K}_{X}$ is analyzed based on the robot translational and rotational displacements. For that matter, two indices $\nu_{p}$ and $\nu_{r}$ were defined to analyze this influence and they are defined as follows:

$$
\nu_{p}=\frac{\left|\delta p_{\mathbf{K}_{C}}-\delta p_{\overline{\mathbf{K}}_{C}}\right|}{\max \left(\delta p_{\mathbf{K}_{C}}, \delta p_{\overline{\mathbf{K}}_{C}}\right)}
$$

and

$$
\nu_{r}=\max \left\{\left|\delta r_{x \mathbf{K}_{C}}-\delta r_{x \overline{\mathbf{K}}_{C}}\right|,\left|\delta r_{y \mathbf{K}_{C}}-\delta r_{y \overline{\mathbf{K}}_{C}}\right|,\left|\delta r_{z \mathbf{K}_{C}}-\delta r_{z \overline{\mathbf{K}}_{C}}\right|\right\}
$$

where $\delta p_{\mathbf{K}_{C}}$ and $\delta p_{\overline{\mathbf{K}}_{C}}$ are the point-displacement of the robot end-effector obtained with Eqs. (7) and (10) assuming that matrix $\mathbf{K}_{C}$ is not null and null, respectively. $\delta r_{x} \mathbf{K}_{C}, \delta r_{y \mathbf{K}_{C}}, \delta r_{z \mathbf{K}_{C}}$ and $\delta r_{x \overline{\mathbf{K}}_{C}}, \delta r_{y \overline{\mathbf{K}}_{C}}, \delta r_{z \overline{\mathbf{K}}_{C}}$ are the small rotations of the robot end-effector about $\mathbf{x}_{0}, \mathbf{y}_{0}$ and $\mathbf{z}_{0}$ axes obtained with Eqs. (7) and (10) assuming that matris

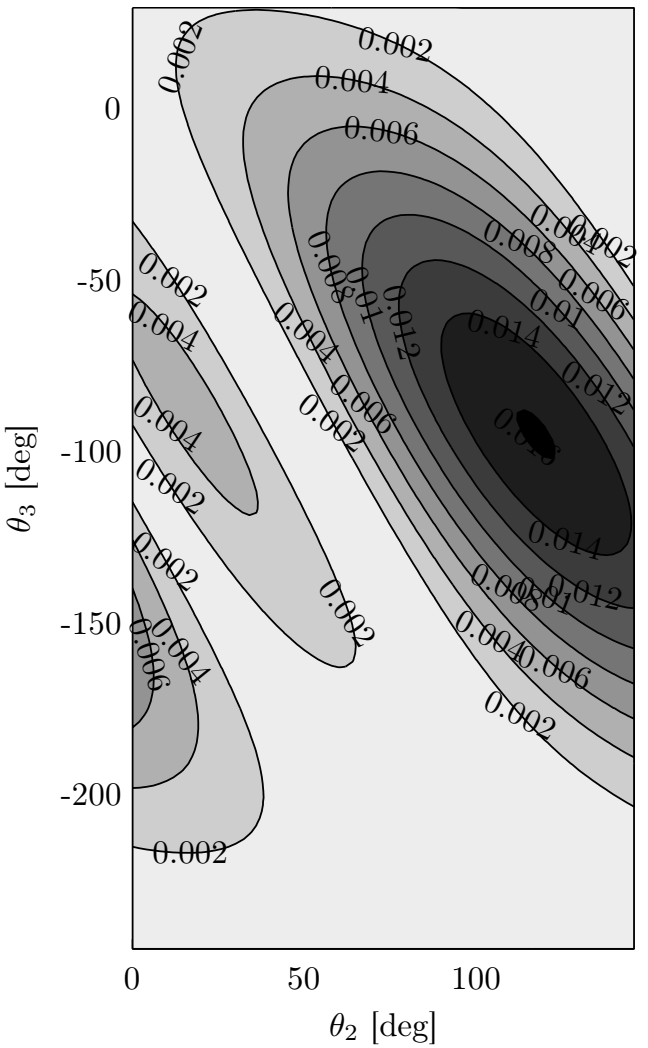

(a)

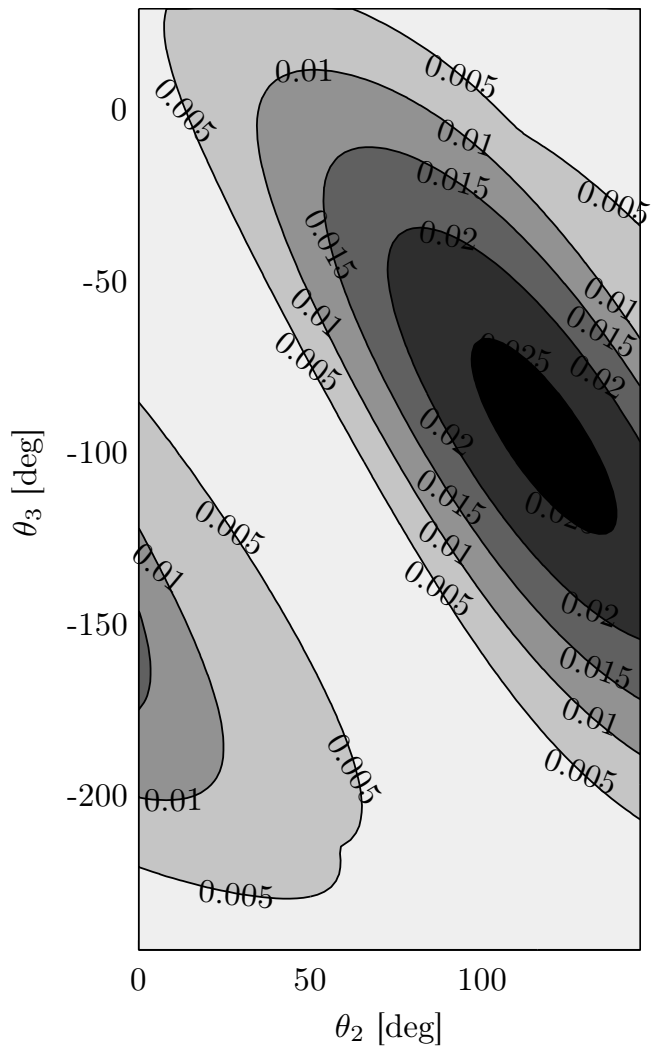

(b)

Figure 4: Isocontours of (a) $\nu_{p}$ and (b) $\nu_{r}$ in the robot joint space $\left(\theta_{2}, \theta_{3}\right)$

Figures 4(a)-(b) illustrate the isocontours of $\nu_{p}$ and $\nu_{r}$ throughout the robot joint space $\left(\theta_{2}, \theta_{3}\right)$. The wrist angles $\theta_{4}, \theta_{5}$ and $\theta_{6}$ are still set to $45^{\circ}$ so that the corresponding wrist configuration is far from singularities, and the wrench is set to $[0,0,-2000 \mathrm{~N}, 200 \mathrm{Nm}, 200 \mathrm{Nm}, 0]$, which can be easily obtained experimentally. Several areas appear, but $\nu_{p}$ and $\nu_{r}$ remain very small as $\nu_{p} \leq 0.016$ and $\nu_{r} \leq 0.025 \mathrm{deg}$ throughout the robot 
joint space. Nevertheless, let $Z_{\nu_{p}}$ and $Z_{\nu_{r}}$ be the zones in which the influence of $\mathbf{K}_{C}$ on $\mathbf{K}_{\theta}$ is a minimum with regard to $\nu_{p}$ and $\nu_{r}$, respectively. To come up with good results, the robot configurations have to be chosen in $Z_{\nu_{p}}$ and $Z_{\nu_{r}}$. As angles $\theta_{2}$ and $\theta_{3}$ have already been fixed to assure a good condition number of the Jacobian matrix, we choose $\theta_{4}$ and $\theta_{5}$ considering this second criterion.

Once the robot configurations are chosen, the wrench exerted on its EE is defined in such a way that its displacement — in terms of translations and rotations — is significant.

\subsection{Evaluation of the joint stiffness values}

From Eq. (10) and assuming that $\mathbf{K}_{C}$ is negligible with respect to $\mathbf{K}_{\theta}$ thanks to an appropriate robot configuration, Eq. (4) can be rewritten as

$$
\boldsymbol{w}=\mathbf{J}^{-T} \mathbf{K}_{\theta} \mathbf{J}^{-1} \delta \mathbf{d}
$$

Hence, the 6-dimensional robot end-effector displacement vector $\delta \mathbf{d}$ takes the form

$$
\delta \mathbf{d}=\mathbf{J K}_{\theta}{ }^{-1} \mathbf{J}^{T} \boldsymbol{w}
$$

Let the joint compliances ${ }^{1}$ be the components of the $6 \times 1$ vector $\mathbf{x}$, namely,

$$
\mathbf{x}=\left[\begin{array}{llllll}
1 / k_{\theta_{1}} & 1 / k_{\theta_{2}} & 1 / k_{\theta_{3}} & 1 / k_{\theta_{4}} & 1 / k_{\theta_{5}} & 1 / k_{\theta_{6}}
\end{array}\right]^{T}
$$

From Eq. (17), it turns out that

$$
\delta \mathbf{d}=\left[\begin{array}{c}
\sum_{j=1}^{6}\left(x_{j} J_{1 j} \sum_{i=1}^{6} J_{i j} w_{i}\right) \\
\vdots \\
\sum_{j=1}^{6}\left(x_{j} J_{6 j} \sum_{i=1}^{6} J_{i j} w_{i}\right)
\end{array}\right]
$$

$x_{j}$ being the $j$ th component of vector $\mathbf{x}$, i.e, $x_{j}=1 / k_{\theta_{j}}, j=1, \ldots, 6$, and $w_{j}$ being the $j$ th component of vector $\boldsymbol{w}$.

By isolating the components of vector $\mathbf{x}$ in Eq. (19), the joint compliances can be expressed with respect to the robot EE displacements as follows:

$$
\mathbf{A x}=\delta \mathbf{d}
$$

A being a $6 \times 6$ matrix taking the form

$$
\mathbf{A}=\left[\begin{array}{ccc}
J_{11} \sum_{i=1}^{6} J_{i 1} w_{i} & \cdots & J_{16} \sum_{i=1}^{6} J_{i 6} w_{i} \\
\vdots & \ddots & \vdots \\
J_{61} \sum_{i=1}^{6} J_{i 1} w_{i} & \cdots & J_{66} \sum_{i=1}^{6} J_{i 6} w_{i}
\end{array}\right]
$$

It is noteworthy that a 6 -dimensional wrench vector, a 6 -dimensional EE displacement vector and a $6 \times 6$ $\mathbf{A}$ matrix are associated with each test. Let $\mathbf{B}_{i}$ and $\mathbf{c}_{i}$ be the matrix $\mathbf{A}$ and the small displacement screw $\delta \mathbf{d}$

\footnotetext{
${ }^{1}$ The compliance stands for the inverse of the stiffness
} 
corresponding to the $i$ th test, respectively. Assuming that $n$ test(s) are used to find $\mathbf{x}$, we obtain:

$$
\mathbf{B x}=\mathbf{c}
$$

with

$$
\mathbf{B}=\left[\begin{array}{c}
\mathbf{B}_{1} \\
\vdots \\
\mathbf{B}_{i} \\
\vdots \\
\mathbf{B}_{n}
\end{array}\right]
$$

and

$$
\mathbf{c}=\left[\begin{array}{c}
\mathbf{c}_{1} \\
\vdots \\
\mathbf{c}_{i} \\
\vdots \\
\mathbf{c}_{n}
\end{array}\right]
$$

It should be noted that the linear-equation system (22) becomes overdetermined when $n>1$ as matrix $\mathbf{B}$ is no longer square, but rectangular. In that case, the joint stiffness values are obtained by minimizing the Euclidean norm of the approximation error $e$ of the overdetermined linear-equation system (22), namely,

$$
\begin{array}{rc}
\operatorname{minimize} & e(\mathbf{x}) \equiv \frac{1}{2}\|\mathbf{B} \mathbf{x}-\mathbf{c}\|_{2}^{2} \\
\text { over } & \mathbf{x}
\end{array}
$$

From [24], the value $\mathbf{x}_{0}$ of $\mathbf{x}$ that minimizes the Euclidean norm of the approximation error $e$ is

$$
\mathbf{x}_{0}=\left(\mathbf{B}^{T} \mathbf{B}\right)^{-1} \mathbf{B}^{T} \mathbf{c}
$$

the matrix coefficient of $\mathbf{c}$ being known as a generalized inverse of $\mathbf{B}$, also known as the left Moore-Penrose generalized inverse of $\mathbf{B}$.

Therefore, several tests can be considered with this approach in order to evaluate the joint stiffness values. Moreover, if all joints are stressed substantially at least once among all the tests, their stiffness value will be accurately evaluated.

\section{Experimental Setup}

As shown in Fig. 5 the experimental setup is composed of a robot, a laser tracker, some retroreflectors and a mass connected to the end-effector by means of a chain and a spring balance. It is used in order to evaluate the wrench exerted on the robot end-effector. The repeatability of the KR240-2 robot is specified to $\pm 0,12 \mathrm{~mm}$ and its workspace radius is equal to $2700 \mathrm{~mm}$.

The measurement system had to guarantee a fast and precise measurement of several points within the robot workspace. For that matter, we used a laser tracker developed by FARO [29]. Its precision depends on the distance between the laser source and the retroreflector and is about $0.03 \mathrm{~mm}$. The system was configured in 


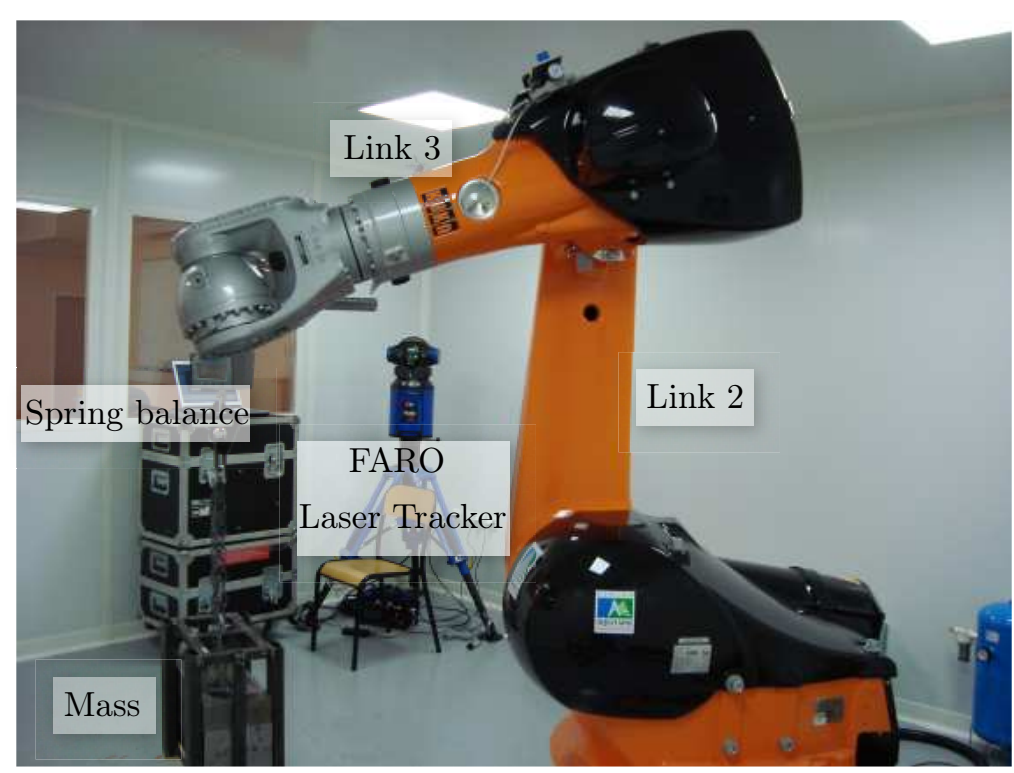

Figure 5: Experimental Setup

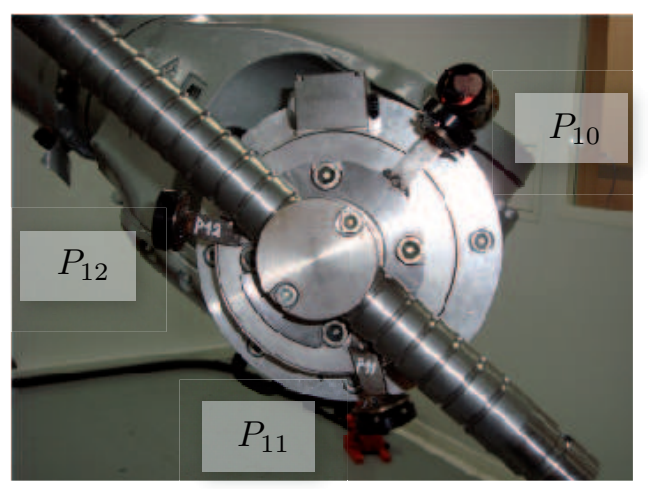

Figure 6: Robot end-effector and location of points $P_{10}, P_{11}$ and $P_{12}$

order to measure all points with respect to the robot base frame.

In order to apply a relevant wrench to the robot EE, we developed a system that can apply a force up to $2500 \mathrm{~N}$ with 250 increments and a moment up to $1250 \mathrm{Nm}$. The joint stiffness values are evaluated from the EE displacements, the robot geometric parameters and the wrench exerted on it. It is necessary to pay attention to the evaluation of the translation and rotational errors of the robot EE. They are evaluated based on the displacements of three points $P_{10}, P_{11}$ and $P_{12}$ attached to the robot EE as shown in Fig. 7 and measured with the FARO Laser Tracker. Frame $\mathcal{F}_{7}$ attached to the robot EE is defined with points $P_{10}, P_{11}$ and $P_{12}$.

Let $\mathcal{F}_{7}$ and $\mathcal{F}_{7}$ ' characterize the pose (position and orientation) of the robot EE before and after the robot deflection due the wrench exerted on it. The transformation between the two foregoing frames characterizes the deflection of the robot (the force sensor deflection, calculated with the wrench and constructor data, is automatically removed from the measured displacement after deformation). Let ${ }^{0} \mathbf{T}_{7}$ and ${ }^{0} \mathbf{T}_{7}$ ' be the homogeneous transformation matrices from the base frame $\mathcal{F}_{0}$ to $\mathcal{F}_{7}$ and from $\mathcal{F}_{0}$ to $\mathcal{F}_{7}$, respectively. Those two matrices, obtained thanks to the coordinates of points $P_{10}, P_{11}$ and $P_{12}$ before and after the robot deflection, take the form:

$$
\begin{aligned}
{ }^{0} \mathbf{T}_{7} & =\left[\begin{array}{cc}
{ }^{0} \mathbf{R}_{7} & { }^{0} \mathbf{p}_{7} \\
\mathbf{0}_{7}^{T} & 1
\end{array}\right] \\
{ }^{0} \mathbf{T}_{7} \prime & =\left[\begin{array}{cc}
{ }^{0} \mathbf{R}_{7^{\prime}} & { }^{0} \mathbf{p}_{7^{\prime}} \\
\mathbf{0}_{7}^{T} & 1
\end{array}\right]
\end{aligned}
$$

From Eq. (27) and Eq. (28), the translational displacement $\delta$ p of the robot EE expressed in $\mathcal{F}_{0}$ is:

$$
\delta \mathbf{p}={ }^{0} \mathbf{p}_{7} \prime-{ }^{0} \mathbf{p}_{7}
$$


and the rotation matrix from $\mathcal{F}_{7}$ to $\mathcal{F}_{7}$ l expressed in $\mathcal{F}_{0}$ is :

$$
\delta \mathbf{R}={ }^{0} \mathbf{R}_{\mathbf{7}}{ }^{7} \mathbf{R}_{\mathbf{0}}
$$

Besides, the wrench exerted on the EE is evaluated by means of the position of the origin of $\mathcal{F}_{7}$, the force direction and the chosen lever arm.

\section{$5 \quad$ Joint Stiffness Values}

\subsection{Robustness of the method}

As the joint stiffness identification requires the measurement of the EE's displacement, the repeatability of this deformation has been checked. The mean uncertainty, i.e. three times the standard deviation, amounts to $1.3 \%$ of the measured deformation, which is acceptable.

In order to analyze the robustness of the method, Eq. (20) has been solved by means of Eq. (26). Figure 7 illustrates joint stiffnesses $k_{\theta_{2}}, k_{\theta_{3}}, k_{\theta_{4}}, k_{\theta_{5}}$ and $k_{\theta_{6}}$ obtained with this method using $n$ test(s), $n=1, \ldots, 23$. Let us note that the $n$ tests are chosen randomly amongst the 23 available ones and a test can not appear two times in a given set.

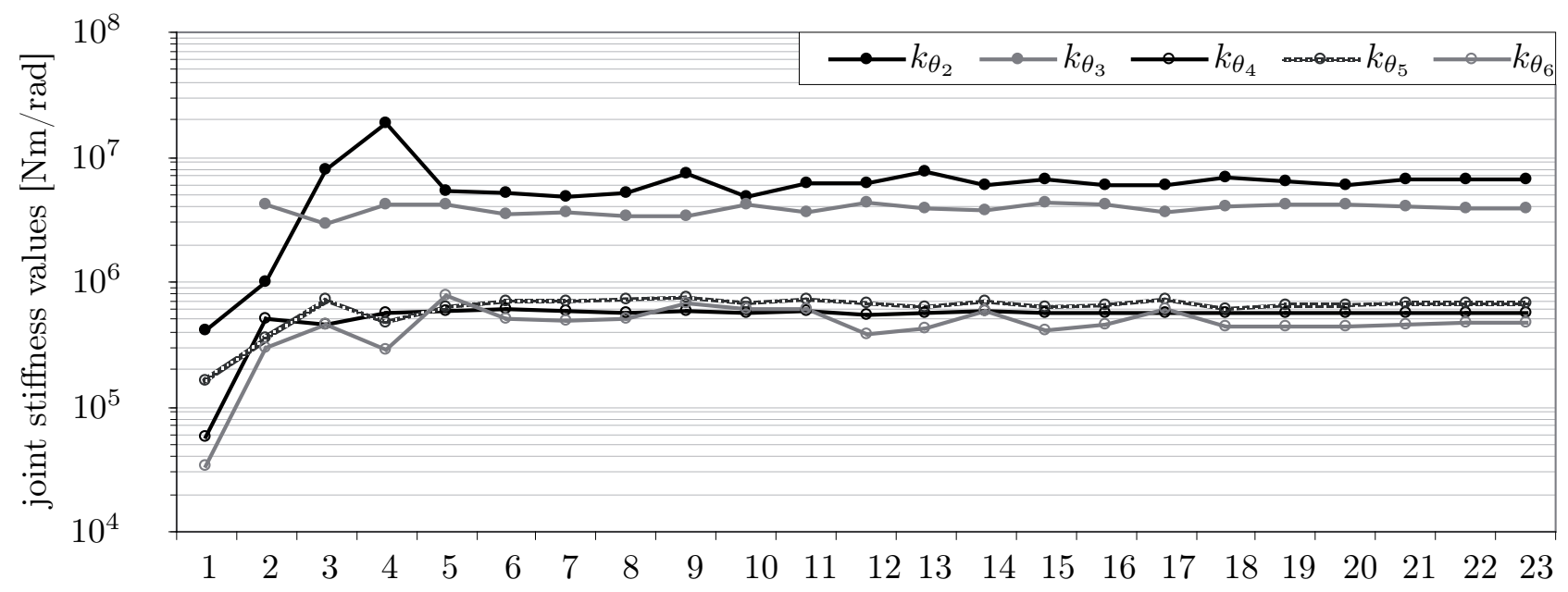

Figure 7: Influence of the number of tests on the joint stiffness values evaluation

We can notice that the larger the size of the set of tests, the better the convergence of joint stiffness values and the more reliable the results. It is apparent that the variations in the obtained joint stiffness values is reasonably small, i.e., the method for the joint stiffness identification is robust, as long as the number of tests considered for their evaluation is higher than six.

\subsection{Sensitivity of the results to measurement errors}

To analyze the sensitivity of the results to measurement errors all parameters defining the 23 tests have been modified by considering errors in the initial parameters. Due to the experimental setup, several sources of errors can be detected: 
- The Faro Tracker Laser uncertainties (about $\pm 0.03 \mathrm{~mm}$ on the distance between the retroreflector and the source)

- The error in the spring balance (about $\pm 0.1 \mathrm{~kg}$ )

- The error in the joint encoders (about $\pm 0.01 \mathrm{deg}$ )

The errors in all the parameters are supposed to be normal. As a consequence, Table 5.2 gives the nominal stiffness value of each joint evaluated by means of 10 tests as well as their error. For instance, the stiffness value of the second joint is equal to $6.610^{6} \mathrm{Nm} / \mathrm{rad}$ while its evaluated error is about $\pm 110^{5} \mathrm{Nm} / \mathrm{rad}$, i.e., $8 \%$ of the nominal stiffness value.

Table 2: Joint stiffness values and errors

\begin{tabular}{cccc}
\hline \hline Joint number & Stiffness values $[\mathrm{Nm} / \mathrm{rad}]$ & Error in $[\mathrm{Nm} / \mathrm{rad}]$ & Percentage of the mean value \\
\hline 1 & $3.810^{6}$ & $\pm 310^{5}$ & $\pm 8 \%$ \\
2 & $6.610^{6}$ & $\pm 5.210^{5}$ & $\pm 8 \%$ \\
3 & $3.910^{6}$ & $\pm 3.710^{5}$ & $\pm 9 \%$ \\
4 & $5.610^{5}$ & $\pm 110^{4}$ & $\pm 2 \%$ \\
5 & $6.610^{5}$ & $\pm 1.410^{4}$ & $\pm 2 \%$ \\
6 & $4.710^{5}$ & $\pm 2.210^{4}$ & $\pm 5 \%$ \\
\hline \hline
\end{tabular}

\subsection{Results}

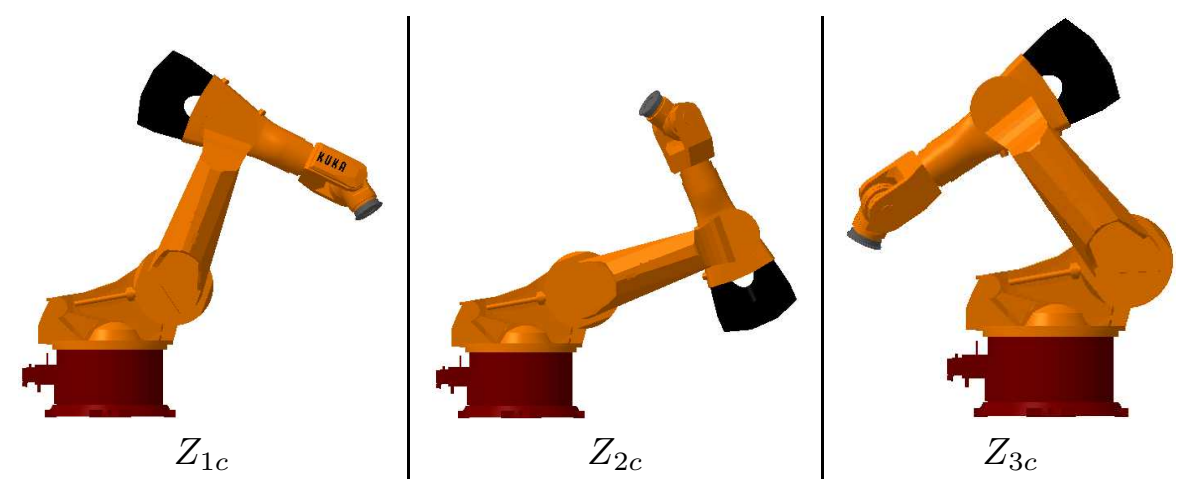

Figure 8: Robot configurations associated with each zone defined in Table 1

Tests are organized with regard to the zones $Z_{1 c}, Z_{2 c}$ and $Z_{3 c}$ defined in Table 1 . Figure 8 illustrates the robot configurations associated with each zone.

Joint stiffness values, reported in Table 5.2 , have been calculated by means of 10 tests realized in $Z_{1 c}, Z_{2 c}$ and $Z_{3 c}$ zones. The model has then been checked on those 10 tests used to determine the joint stiffness values, on 13 other tests done in $Z_{1 c}, Z_{2 c}$ and $Z_{3 c}$ zones but that have not been used to identify the joint stiffness values, and on 2 last tests that have been done in non-optimal areas with respect to the criteria presented in Section 3. It is noteworthy that $\mathbf{K}_{\mathbf{C}}$ matrix is taken into account in the stiffness model to carry out checks.

Figure 9 depicts the calculated and measured displacements of the EE. Validation is realized with the tests used for the joint stiffness identification (Fig. 9-(a)) and with the other tests (Fig. 9-(b)). The circle 


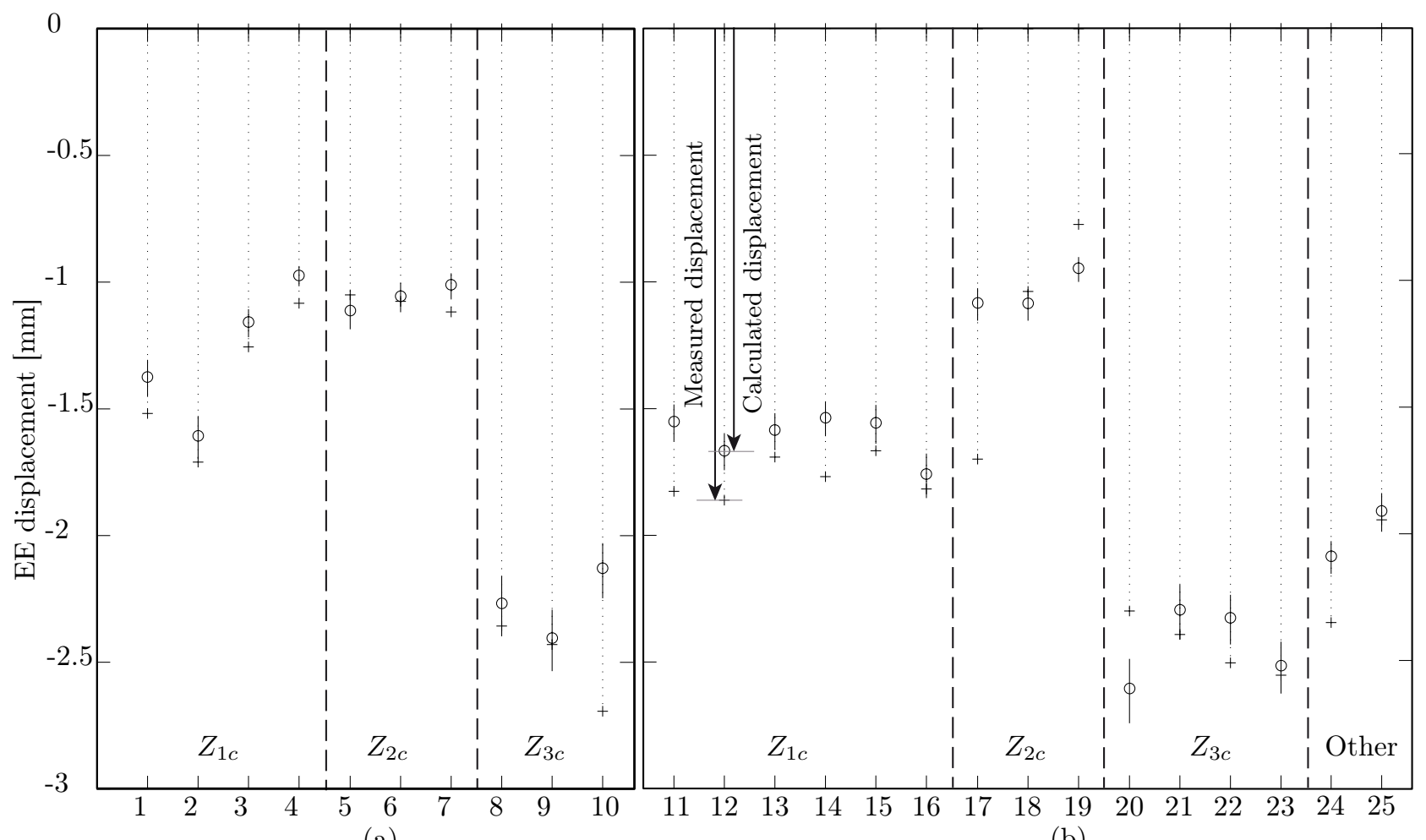

(a)

(b)

Figure 9: Theoretical and measured robot EE translational displacements: (a) Validation with the tests used for the joint stiffness identification; (b) Validation with the other tests

denotes the calculated, i.e., theoretical, displacement of the robot EE while the cross denotes its measured displacement. Moreover, the line segments around the circles depict the error in the calculated robot EE displacement due to measurement uncertainties. The longer the segment, the higher the error in the calculated robot EE displacement. It appears that errors in the calculated robot EE displacement are quite smaller than the displacement itself, namely, the joint stiffnesses identification is robust with respect to measurement errors. Over the 25 tests, the average difference between the theoretical and measured displacements is about $0.1 \mathrm{~mm}$, and the maximum gap is equal to $0.6 \mathrm{~mm}$. The method can predict about $80 \%$ of the displacement of the EE. In order to explain the un-corrected $20 \%$ of the displacement of the EE, the link stiffnesses have been assessed. As links 2 and 3 are the largest ones, the main source of the robot compliance, except the joint compliance, should be their flexibility. Consequently, their deformations under several loadings have been measured and compared to the EE displacement. Results show that deformations of link 3 are responsible for a maximum of $4.8 \%$ of the EE displacement (when the link is horizontal and the load on the EE is at the maximum). As link 2 is far form the EE, the influence of its deformations on the latter is more important: maximum $21 \%$. So a maximum of $25 \%$ of the EE displacement, depending on the robot configuration and applied load, can be due to link flexibilities. Therefore, it is a limit of the identification method proposed in this paper.

Figures 10 to 11 illustrate the calculated and measured rotations of the robot EE about $\mathbf{x}, \mathbf{y}$ and $\mathbf{z}$ axes and expressed in $\mathcal{F}_{0}$. The circle denotes the calculated, i.e., theoretical, rotation of the robot EE while the cross denotes its measured rotation. Over the 25 tests used to check the model, the average difference between the theoretical and measured rotations is about 0.005 degree around $\mathbf{x}$ and $\mathbf{y}$, and about 0.01 degree around $\mathbf{z}$. This gap is due to the fact that the sixth actuated joint was not as stressed as the fourth and fifth ones amongst all 


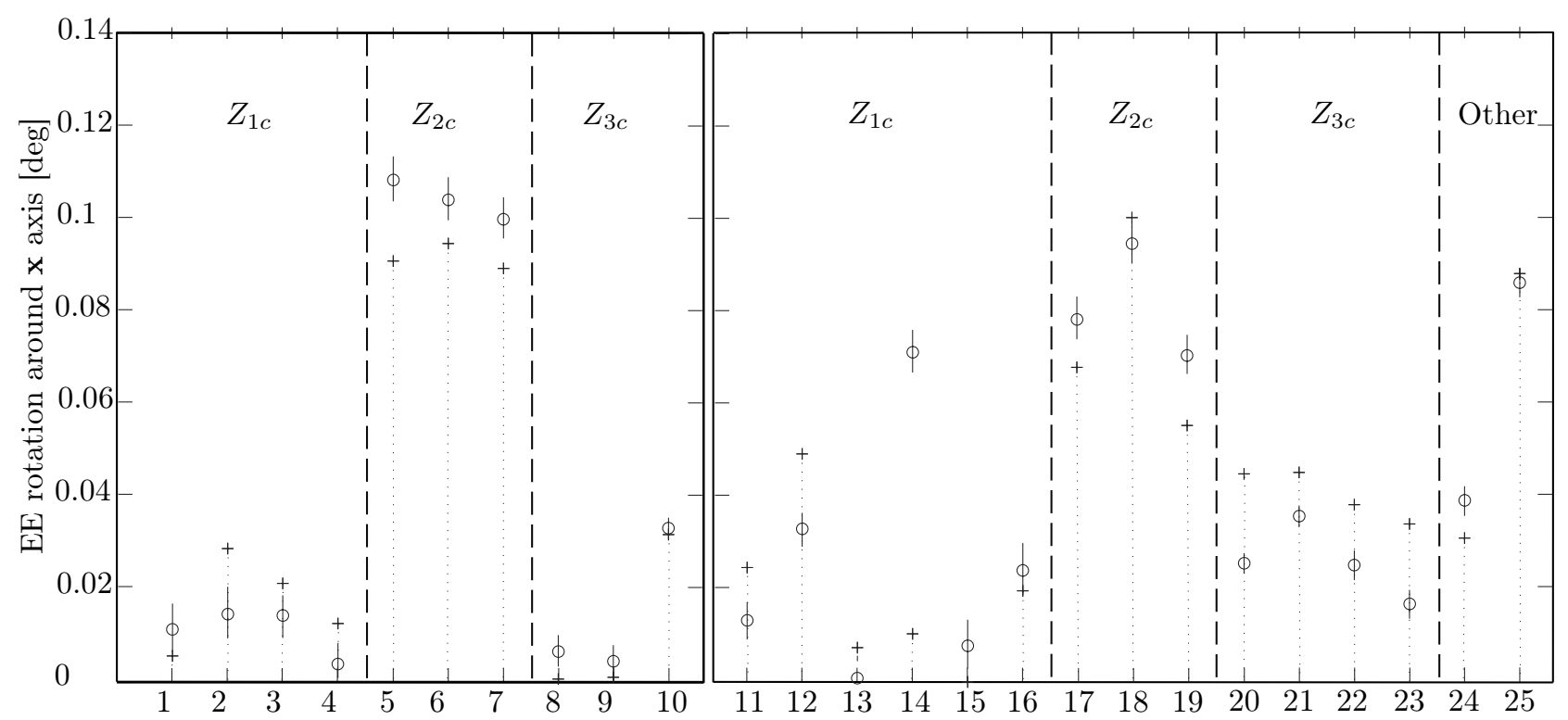

(a)

(b)

Figure 10: Calculated and measured rotations of the EE expressed around $\mathbf{x}$ axis of $\mathcal{F}_{0}$ : (a) Validation with the tests used for the joint stiffness identification; (b) Validation with the other tests

the tests.

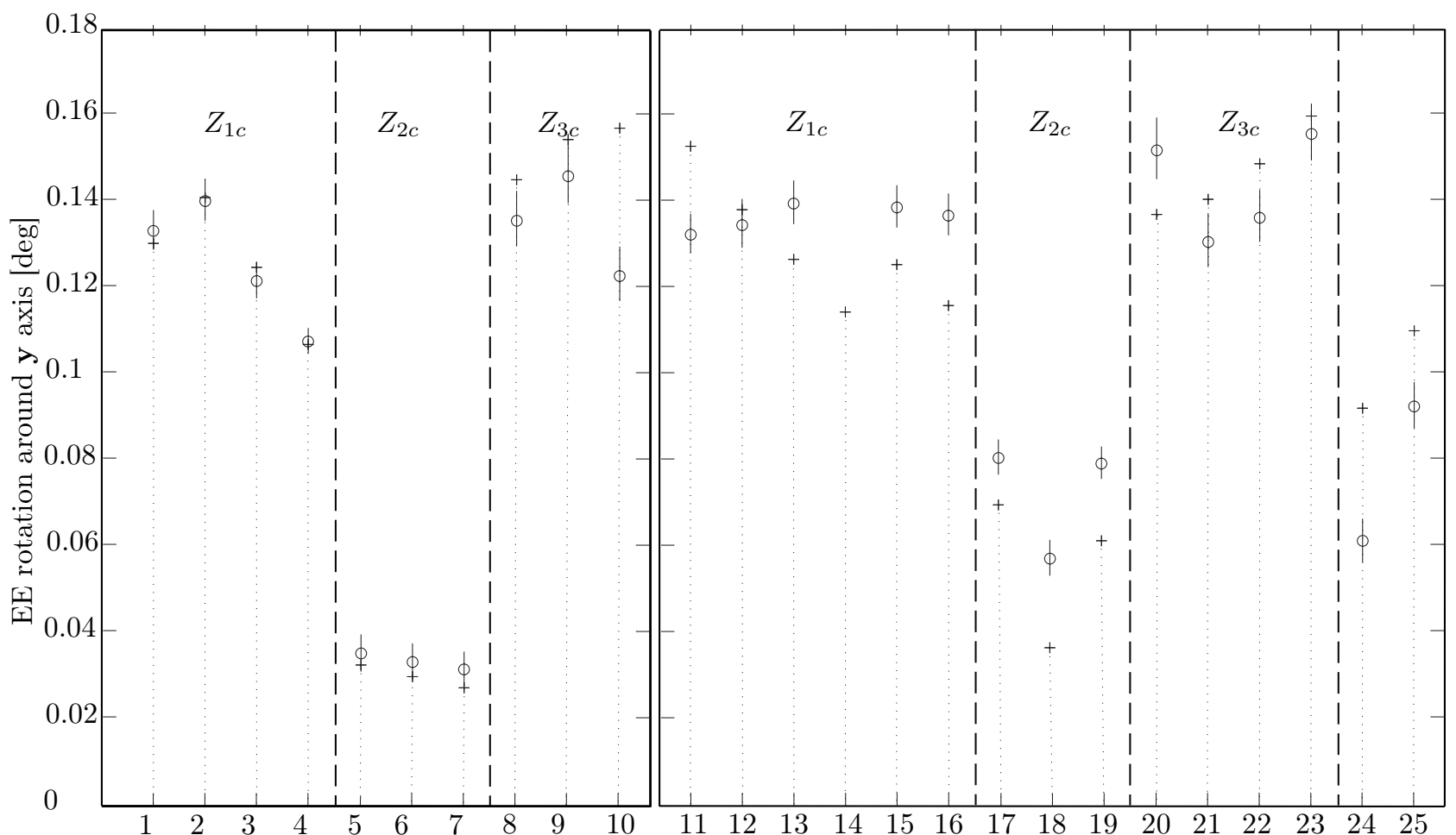

(a)

(b)

Figure 11: Calculated and measured rotations of the EE expressed around $\mathbf{y}$ axis of $\mathcal{F}_{0}$ : (a) Validation with the tests used for the joint stiffness identification; (b) Validation with the other tests 


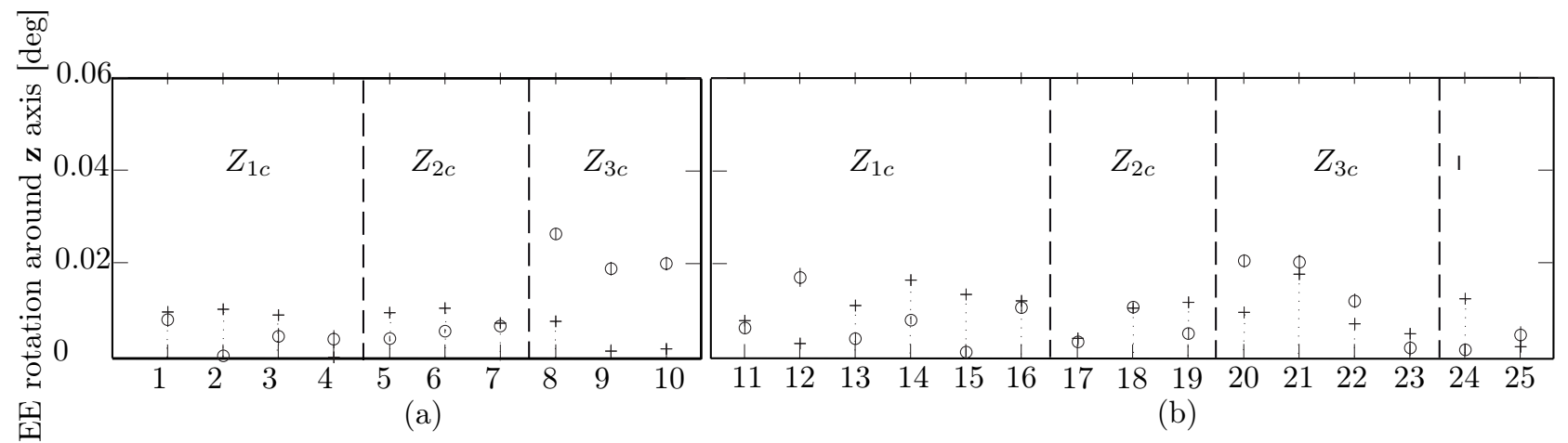

Figure 12: Calculated and measured rotations of the EE expressed around $\mathbf{z}$ axis of $\mathcal{F}_{0}$ : (a) Validation with the tests used for the joint stiffness identification; (b) Validation with the other tests

\section{Conclusions}

This paper dealt with a new methodology for joint stiffness identification of industrial serial robots. This methodology aims at evaluating the joint stiffness values responsible for both translational and rotational displacements of the robot end-effector subject to an external wrench. As a result, the coupling between the robot end-effector translational and rotational displacements is considered and the joint stiffness values of both the robot translational part and wrist can be identified. The Kuka KR240-2 robot was used as an illustrative example throughout the paper. First, its kinematic model was obtained in order to determine the optimal robot configurations according to the condition number of its kinematic Jacobian matrix. Then, its stiffness model was developed by means of its Cartesian stiffness matrix $\mathbf{K}_{X}$ and its Complementary stiffness matrix $\mathbf{K}_{C}$. As the stiffness model and the identification of the joint stiffness values turn to be simple when the latter is negligible with respect to the former, the robot configurations that minimize the influence of $\mathbf{K}_{C}$ on $\mathbf{K}_{X}$ were determined. The experimental setup and the experimental procedure were also presented. It is noteworthy that the links and transmissions of the robot were supposed to be quite stiffer than the joints and not known as it is usually the case for industrial robots. As a consequence, a good approximation of the real joint stiffness values was obtained with the proposed methodology. The robustness of the latter and the sensitivity of the results to measurement errors and to the number of experimental tests were also analyzed. Finally, the proposed methodology will be improved in a future work to identify the link stiffnesses in addition to the joint stiffnesses of industrial robots.

\section{Acknowledgments}

The authors would like to acknowledge the financial support of the French "Agence Nationale de la Recherche" (Project ANR-2010-SEGI-003-02-COROUSSO). The FARO company is also dutifully acknowledged for its great help with the measurements.

\section{References}

[1] Zha, Xuan F. (2002). "Optimal Pose Trajectory Planning for Robot Manipulators," Mechanism and Machine Theory, 37, pp. 1063-1086. 
[2] Kim, T. and Sarma, S-E. (2002). "Toolpath Generation along directions of Maximum Kinematic Performance; a first cut at Machine-Optimal Paths," Computer-Aided Design, 34, pp. 453-468.

[3] Matsuoka, S.-I.,Shimizu, K.,Yamazaki, N. and Oki, Y. (1999). "High-Speed End Milling of an Articulated Robot and its Characteristics," Elsevier, Journal of Materials Processing Technology, 95, pp. 83-89.

[4] Pan, Z., Zhang, H., Zhu, Z. and Wang, J. (2006). "Chatter Analysis of Robotic Machining Process," Journal of Materials Processing Technology, 173, pp. 301-309.

[5] Nagata, F.,Hase, T.,Haga, Z.,Omota, M. and Watanabe, K. (2007). "CAD/CAM-based Position/Force Controller for a Mold Polishing Robot," Elsevier, Mechatronics, 17, pp. 207-216.

[6] Zhang, H., Hang, H., Wang, J., Zhang, G., Gan, Z., Pan, Z., Cui, H. and Zhu, Z. (2005). "Machining with Flexible Manipulator: Toward Improving Robotic Machining Performance," Proceedings of the 2005 IEEE/ASME International Conference on Advanced Intelligent Mechatronics, Monterey, California, USA, 24-28 July.

[7] Nawratil, G. (2007). "New Performance Indices for 6R Robots," Mechanism and Machine Theory, 42, pp. 1499-1511.

[8] Kucuk, S. and Bingul, Z. (2006). "Comparative Study of Performance Indices for Fundamental Robot Manipulators," Robotics and Autonomous Systems, 54, pp. 567-573.

[9] Mansouri, I. and Ouali, M.(2009). "A new homogeneous manipulability measure of robot manipulators, based on power concept," Mechatronics, 19, pp. 927-944.

[10] Kim, B.H., Yi, B.J., Oh, S.R. and Suh, I.H. (2004). "Non-dimensionalized performance indices based optimal grasping for multi-fingered hands," Mechatronics, 14, pp. 255-280.

[11] Dumas, C., Caro, S., Garnier, S. and Furet, B. (2011). "Joint Stiffness Identification of Six-revolute Industrial Serial Robots," Robotics and Computer Integrated Manufacturing, 27(4), pp. 881-888.

[12] Pashkevich, A., Chablat, D. and Wenger, P. (2009). "Stiffness Analysis of Overconstrained Parallel Manipulators," Mechanism and Machine Theory, 44, pp. 966-982.

[13] Östring, M., Gunnnarsson, S. and Norrlf, M. (2009). "Closed-loop Identification of an Industrial Robot Containing Flexibilities," Control Engineering Practice, 11, pp. 291-300.

[14] Chen, S.-F. (2003). "The 6x6 Stiffness Formulation and Transformation of Serial Manipulators via the CCT Theory," IEEE International Conference on Robotics \& Automation, Taiwan.

[15] Alici, G. and Shirinzadeh, B. (2005). "Enhanced Stiffness Modeling, Identification and Characterization for Robot Manipulators," IEEE Transactions on Robotics, 21(4), pp. 554-564.

[16] Chen, S.-F. and Kao, I. (2000). "Conservative Congruence Transformation for Joint and Cartesian Stiffness Matrices of Robotics Hands and Fingers," The International Journal of Robotics Research, 19(9), pp. 835847.

[17] Abele, E., Weigold, M. and Rothenbcher, S. (2007). "Modeling and Identification of an Industrial Robot for Machining Applications," Elsevier, Annals of the CIRP, 56/1/2007. 
[18] Pham, M. T., Gautier, M. and Poignet, P. (2001). "Identification of Joint Stiffness with Bandpass Filtering," Proceedings of the 2001 IEEE International Conference on Robotics \& Automation Seoul, Korea, 21-26 May.

[19] Khalil, W. and Dombre, E. (2002). "Modeling, Identification and Control of Robots," Hermes Science Publications.

[20] SYMORO+. The software package SYMORO+, "SYmbolic MOdeling of RObots," is the outcome of the research activity of the robotics team of the IRCCyN in the field of robot modeling.

[21] Merlet, J.P. (2006). "Jacobian, Manipulability, Condition Number, and Accuracy of Parallel Robots," ASME Journal of Mechanical Design, 128, pp. 199-205.

[22] Caro, S., Binaud, N. and Wenger, P. (2009). "Sensitivity Analysis of 3-RPR Planar Parallel Manipulators," ASME Journal of Mechanical Design, 131, pp. 121005-1-121005-13.

[23] Angeles, J. (2007). Fundamentals of Robotic Mechanical Systems Theory, Methods, and Algorithms, Third Edition, Springer, New York (first edition published in 1997).

[24] Golub, G.H. and Van Loan, C.F. (1989). Matrix Computations, The Johns Hopkins University Press, Baltimore.

[25] Li, Z. (1990). "Geometrical Consideration of Robot Kinematics," The International Journal of Robotics and Automation, 5(3), pp. 139-145.

[26] Paden, B. and Sastry, S. (1988). "Optimal Kinematic Design of 6R Manipulator," The International Journal of Robotics Research, 7(2), pp. 43-61.

[27] Ranjbaran, F., Angeles, J., Gonzalez-Palacios, M. A. and Patel, R. V. (1995). "The Mechanical Design of a Seven-Axes Manipulator with Kinematic Isotropy," ASME Journal of Intelligent and Robotic Systems, 14(1), pp. 21-41.

[28] Khan, W.A. and Angeles, J. (2006). "The Kinetostatic Optimization of Robotic Manipulators: The Inverse and the Direct Problems," ASME Journal of Mechanical Design, 128, pp. 168-178.

[29] www.faro.com

\section{A Kinematic Jacobian Matrix J}

The DHm parameters of the robot are given in Table 3.

Table 3: DHm parameters of the Kuka KR240-2 robot

\begin{tabular}{ccccc}
\hline \hline$j$ & $\alpha_{j}$ & $d_{j}$ & $\theta_{j}$ & $r_{j}$ \\
\hline 1 & 0 & 0 & $\theta_{1}$ & 0 \\
2 & $\pi / 2$ & $d_{2}$ & $\theta_{2}$ & 0 \\
3 & 0 & $d_{3}$ & $\theta_{3}$ & 0 \\
4 & $-\pi / 2$ & $d_{4}$ & $\theta_{4}$ & $R L_{4}$ \\
5 & $\pi / 2$ & 0 & $\theta_{5}$ & 0 \\
6 & $-\pi / 2$ & 0 & $\theta_{6}$ & $R L_{6}$ \\
\hline \hline
\end{tabular}

Those parameters are defined in [19]: 
- $\alpha_{j}$ : angle between axis $\mathbf{z}_{j-1}$ and $\mathbf{z}_{j}$ around $\mathbf{x}_{j-1}$

- $d_{j}$ : distance between axis $\mathbf{z}_{j-1}$ and $\mathbf{z}_{j}$ along $\mathbf{x}_{j-1}$

- $\theta_{j}$ : angle between axis $\mathbf{x}_{j-1}$ and $\mathbf{x}_{j}$ around $\mathbf{z}_{j}$

- $r_{j}$ : distance between axis $\mathbf{x}_{j-1}$ and $\mathbf{x}_{j}$ along $\mathbf{z}_{j}$

The kinematic Jacobian Matrix $\mathbf{J}$ of the Kuka KR240-2 robot is expressed as follows:

$$
\mathbf{J}=\left[\begin{array}{cccccc}
J_{11} & J_{12} & J_{13} & J_{14} & J_{15} & J_{16} \\
J_{21} & J_{22} & J_{23} & J_{24} & J_{25} & J_{26} \\
J_{31} & J_{32} & J_{33} & J_{34} & J_{35} & J_{36} \\
J_{41} & J_{42} & J_{43} & J_{44} & J_{45} & J_{46} \\
J_{51} & J_{52} & J_{53} & J_{54} & J_{55} & J_{56} \\
J_{61} & J_{62} & J_{63} & J_{64} & J_{65} & J_{66}
\end{array}\right]
$$

with

$$
\begin{aligned}
& J_{11}=-d_{2} \sin \theta_{1}-d_{3} \cos \theta_{2} \sin \theta_{1}-d_{4} \cos \left(\theta_{2}+\theta_{3}\right) \sin \theta_{1}+R L_{4} \sin \theta_{1} \sin \left(\theta_{2}+\theta_{3}\right) \\
& +R L_{6} \cos \theta_{5} \sin \theta_{1} \sin \left(\theta_{2}+\theta_{3}\right)+R L_{6} \cos \left(\theta_{2}+\theta_{3}\right) \cos \theta_{4} \sin \theta_{1} \sin \theta_{5}+R L_{6} \cos \theta_{1} \sin \theta_{4} \sin \theta_{5} \\
& J_{12}=-R L_{4} \cos \theta_{1} \cos \left(\theta_{2}+\theta_{3}\right)-R L_{6} \cos \theta_{1} \cos \left(\theta_{2}+\theta_{3}\right) \cos \theta_{5}-d_{3} \cos \theta_{1} \sin \theta_{2} \\
& -d_{4} \cos \theta_{1} \sin \left(\theta_{2}+\theta_{3}\right)+R L_{6} \cos \theta_{1} \cos \theta_{4} \sin \left(\theta_{2}+\theta_{3}\right) \sin \theta_{5} \\
& J_{13}=-R L_{4} \cos \theta_{1} \cos \left(\theta_{2}+\theta_{3}\right)-R L_{6} \cos \theta_{1} \cos \left(\theta_{2}+\theta_{3}\right) \cos \theta_{5}-d_{4} \cos \theta_{1} \sin \left(\theta_{2}+\theta_{3}\right) \\
& +R L_{6} \cos \theta_{1} \cos \theta_{4} \sin \left(\theta_{2}+\theta_{3}\right) \sin \theta_{5} \\
& J_{14}=R L_{6} \cos \theta_{4} \sin \theta_{1} \sin \theta_{5}+R L_{6} \cos \theta_{1} \cos \left(\theta_{2}+\theta_{3}\right) \sin \theta_{4} \sin \theta_{5} \\
& J_{15}=-R L_{6} \cos \theta_{1} \cos \left(\theta_{2}+\theta_{3}\right) \cos \theta_{4} \cos \theta_{5}+R L_{6} \cos \theta_{5} \sin \theta_{1} \sin \theta_{4}+R L_{6} \cos \theta_{1} \sin \left(\theta_{2}+\theta_{3}\right) \sin \theta_{5} \\
& J_{16}=0 \\
& J_{21}=d_{2} \cos \theta_{1}+d_{3} \cos \theta_{1} \cos \theta_{2}+d_{4} \cos \theta_{1} \cos \left(\theta_{2}+\theta_{3}\right)-R L_{4} \cos \theta_{1} \sin \left(\theta_{2}+\theta_{3}\right)-R L_{6} \cos \theta_{1} \cos \theta_{5} \sin \left(\theta_{2}+\theta_{3}\right) \\
& -R L_{6} \cos \theta_{1} \cos \left(\theta_{2}+\theta_{3}\right) \cos \theta_{4} \sin \left(\theta_{5}\right)+R L_{6} \sin \theta_{1} \sin \theta_{4} \sin \theta_{5} \\
& J_{22}=-R L_{4} \cos \left(\theta_{2}+\theta_{3}\right) \sin \theta_{1}-R L_{6} \cos \left(\theta_{2}+\theta_{3}\right) \cos \theta_{5} \sin \theta_{1}-d_{3} \sin \theta_{1} \sin \theta_{2}-d_{4} \sin \theta_{1} \sin \left(\theta_{2}+\theta_{3}\right) \\
& +R L_{6} \cos \theta_{4} \sin \theta_{1} \sin \left(\theta_{2}+\theta_{3}\right) \sin \theta_{5} \\
& J_{23}=-R L_{4} \cos \left(\theta_{2}+\theta_{3}\right) \sin \theta_{1}-R L_{6} \cos \left(\theta_{2}+\theta_{3}\right) \cos \theta_{5} \sin \theta_{1}-d_{4} \sin \theta_{1} \sin \left(\theta_{2}+\theta_{3}\right) \\
& +R L_{6} \cos \theta_{4} \sin \theta_{1} \sin \left(\theta_{2}+\theta_{3}\right) \sin \theta_{5} \\
& J_{24}=-R L_{6} \cos \theta_{1} \cos \theta_{4} \sin \theta_{5}+R L_{6} \cos \left(\theta_{2}+\theta_{3}\right) \sin \theta_{1} \sin \theta_{4} \sin \theta_{5} \\
& J_{25}=-R L_{6} \cos \left(\theta_{2}+\theta_{3}\right) \cos \theta_{4} \cos \theta_{5} \sin \theta_{1}-R L_{6} \cos \theta_{1} \cos \theta_{5} \sin \theta_{4}+R L_{6} \sin \theta_{1} \sin \left(\theta_{2}+\theta_{3}\right) \sin \theta_{5} \\
& J_{26}=0 \\
& J_{31}=0 \\
& J_{32}=d_{3} \cos \theta_{2}+d_{4} \cos \left(\theta_{2}+\theta_{3}\right)-R L_{4} \sin \left(\theta_{2}+\theta_{3}\right)-R L_{6} \cos \theta_{5} \sin \left(\theta_{2}+\theta_{3}\right) \\
& -R L_{6} \cos \left(\theta_{2}+\theta_{3}\right) \cos \theta_{4} \sin \theta_{5} d_{4} \cos \left(\theta_{2}+\theta_{3}\right)-R L_{4} \sin \left(\theta_{2}+\theta_{3}\right)-R L_{6} \cos \theta_{5} \sin \left(\theta_{2}+\theta_{3}\right) \\
& -R L_{6} \cos \left(\theta_{2}+\theta_{3}\right) \cos \theta_{4} \sin \theta_{5}
\end{aligned}
$$




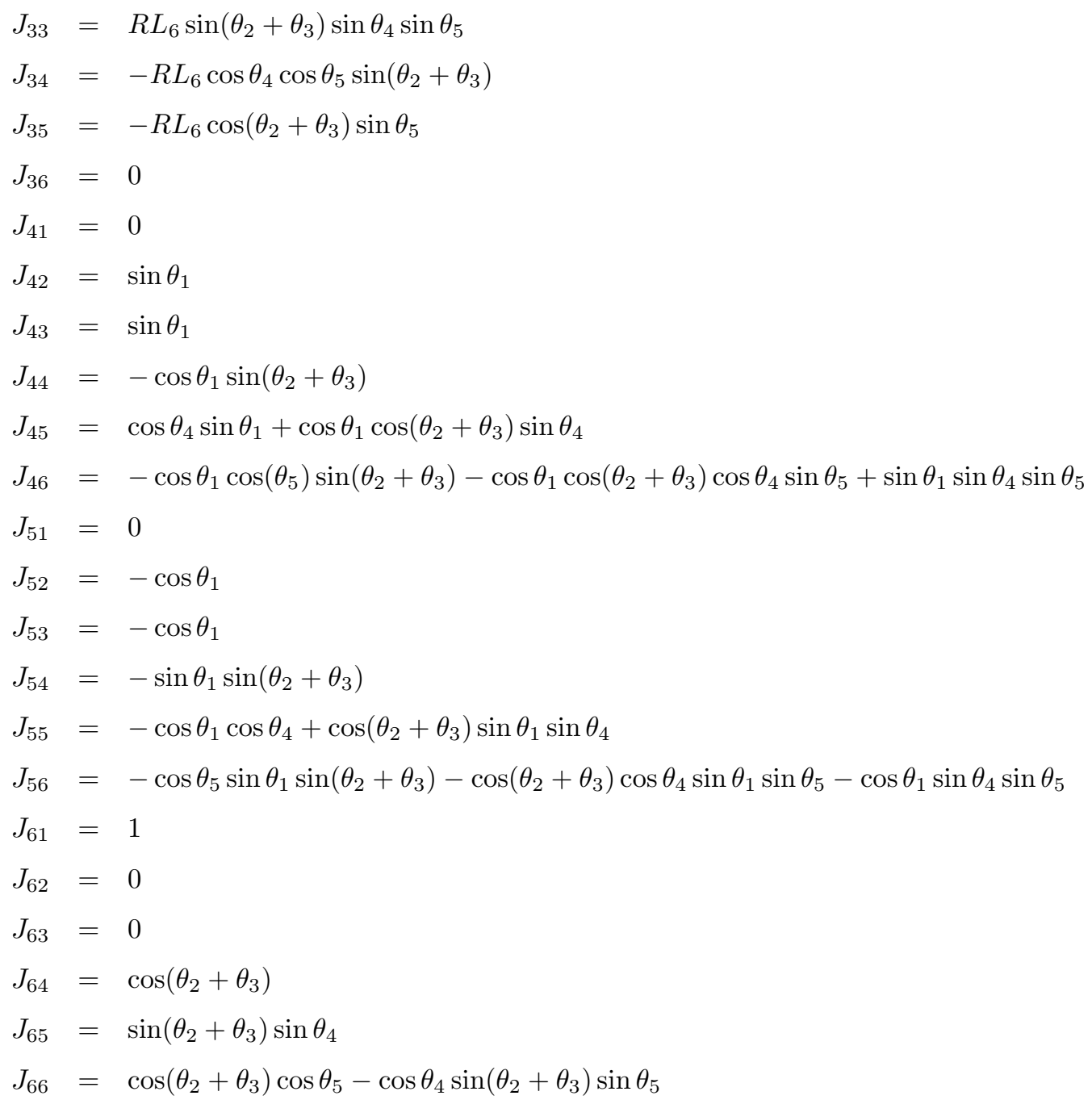

